\title{
Comparison of formulations for representing the fluid-solid coupling using the spectral element method
}

\author{
J. Cao ${ }^{1 *}$, R. Brossier ${ }^{1}$, L. Métivier ${ }^{1,2}$ \\ ${ }^{1}$ Univ. Grenoble Alpes, ISTerre, F-38058 Grenoble, France \\ ${ }^{2}$ CNRS, Univ. Grenoble Alpes, LJK, F-38058 Grenoble, France
}

Wednesday $15^{\text {th }}$ January, 2020

\begin{abstract}
Main objectives This study considers applying the spectral element method to seismic wave modeling in fluid-solid coupled media. It concentrates on the coupling between two wave equations: the acousticwave equation for the fluid domain and the elastic-wave equation for the solid domain. Existing formulations for representing the fluid-solid coupling are summarized and compared to present their pros and cons in the spectral element method.
\end{abstract}

New aspects covered High-order spectral element method is applied to simulate seismic waves in marine environments with complex fluid-solid interface. Pros and cons of existing formulations for fluid-solid coupling are presented within the framework of spectral element method.

\section{Summary}

We consider modeling seismic wave propagation in fluid-solid coupled media, in the perspective of seismic exploration in marine environments. The spectral element method is used for the spatial discretization due to its high accuracy in relatively smooth media and high computational efficiency through a domain-decomposition based parallelization. During the implementation, fluid and solid domains are divided explicitly and handled with the acoustic-wave and elastic-wave equation, respectively. The mutual interaction between these two wave equations is modeled by coupling conditions at the fluid-solid interface. The weak forms of 4 existing coupling formulations in terms of pressure, velocity potential, displacement potential and displacement are constructed as $P-\mathbf{u}_{s}, \phi-\mathbf{u}_{s}, \varphi-\mathbf{u}_{s}$, and $\mathbf{u}_{\mathbf{f}}-\mathbf{u}_{\mathrm{s}}$ system, respectively. Several benchmark tests are carried out to analyze their pros and cons in the spectral element method, from the aspects of complexity, accuracy and computational efficiency. 


\section{Introduction}

In marine seismic exploration, an accurate modeling of seismic wave propagation would require to be implemented in media which contain both fluid and solid regions, in particular for the inversion of ocean bottom-recorded data where the horizontal components show significant elastic effects from $\mathrm{P}$ to $\mathrm{S}$ conversions. Although some numerical methods designed by finite-difference discretization can deal with this fluid-solid contrast explicitly or implicitly, they have not presented superior accuracy and flexibility compared with some weak-form based methods, such as the spectral element method (SEM). The SEM has been shown to be efficient for its high accuracy in relatively smooth media and high computational efficiency through a domain-decomposition based parallelization (Komatitsch, 1997, Peter et al., 2011).

As pointed out by Komatitsch et al. (2000), a classical SEM formulated using elastic-wave equation does not satisfy the fluid-solid boundary condition correctly and will introduce strong artifacts in the fluid region. Thus, a conventional way in SEM is to divide the model into solid and fluid domains. The acoustic-wave equation is used to describe the wave propagation in the fluid domain, while the elasticwave equation models elastic vibrations in the solid domain. To account for the mutual interaction between the two domains, coupling conditions need to be applied at the fluid-solid interface. In the solid domain, the elastic-wave equation is commonly formulated in terms of displacement. The fluid, however, can be modeled in terms of pressure, velocity potential, displacement potential or displacement (Everstine, 1997), yielding 4 formulations for fluid-solid coupled modeling (Feng, 2000; Komatitsch et al., 2000, Chaljub and Valette, 2004; Ross et al., 2009).

Here we conduct a comparison study on the SEM implementation of these 4 formulations for fluid-solid coupled modeling. We show how these coupling formulations can be expressed within the framework of SEM in detail. As a comparison, several benchmark tests are carried out to reveal their pros and cons in terms of accuracy and computational efficiency. We conclude that best trade-off is achieved by the displacement potential formulation in the fluid domain. We embed it in our SEM46 code (Trinh et al., 2019) and show a preliminary 3D fluid-solid modeling result, in the perspective to extend its application in marine seismic exploration.

\section{Formulate the fluid-solid coupled problem in SEM}

In the solid region, the isotropic elastic-wave equation considered in this study can be written as

$$
\rho_{s} \ddot{\mathbf{u}}_{s}=\nabla \cdot \boldsymbol{\sigma}+\mathbf{f}_{s}, \quad \boldsymbol{\sigma}=\lambda \operatorname{tr}(\boldsymbol{\varepsilon}) \mathbf{I}+2 \mu \boldsymbol{\varepsilon}, \quad \boldsymbol{\varepsilon}=\frac{1}{2}\left[\nabla \mathbf{u}_{s}+\left(\nabla \mathbf{u}_{s}\right)^{T}\right],
$$

where $\mathbf{u}_{s}$ and $\mathbf{f}_{s}$ are the displacement and force vector, respectively, $\boldsymbol{\sigma}$ is the stress tensor, $\boldsymbol{\varepsilon}$ is the strain tensor, $\lambda$ and $\mu$ are the Lamé parameters, $\rho_{s}$ is the solid density, and $\mathbf{I}$ is the identity tensor.

Assuming an irrotational and inviscid fluid, its wavefield is governed by the following conservation and dynamic equations:

$$
\rho_{f} \ddot{\mathbf{u}}_{f}+\nabla P=\mathbf{f}_{f}, \quad \dot{P}+\kappa \nabla \cdot \dot{\mathbf{u}}_{f}=0,
$$

where $\mathbf{u}_{f}$ and $\mathbf{f}_{f}$ are the displacement and force vector in the fluid domain, respectively, $P$ is the pressure, $\rho_{f}$ is the fluid density, and $\kappa$ is the bulk modulus of the fluid. By substitution and elimination, Eq. (2) can be rewritten in terms of fluid pressure $(P)$, displacement potential $(\varphi)$, velocity potential $(\phi)$ or displacement $\left(\mathbf{u}_{f}\right)$, respectively:

$$
\begin{gathered}
\ddot{P}+\kappa \nabla \cdot\left(-\frac{1}{\rho_{f}} \nabla P\right)=P_{f}, \quad \ddot{\varphi}-\kappa \nabla \cdot\left(\frac{1}{\rho_{f}} \nabla \varphi\right)=\iint-P_{f} d t d t, \\
\ddot{\phi}-\frac{\kappa}{\rho_{f}} \nabla \cdot \nabla \phi=\int-\frac{1}{\rho_{f}} P_{f} d t, \quad \rho_{f} \ddot{\mathbf{u}}_{f}-\nabla\left(\kappa \nabla \cdot \mathbf{u}_{f}\right)=\mathbf{f}_{f} .
\end{gathered}
$$

Here, $P_{f}$ is the pressure source related to the force vector $\left(\mathbf{f}_{f}\right)$, and the velocity potential $(\phi)$ and displacement potential $(\varphi)$ are defined as

$$
\dot{\mathbf{u}}_{f}:=\nabla \phi, \quad \mathbf{u}_{f}:=\frac{1}{\rho} \nabla \varphi
$$

and their relationship with pressure $(P)$ are

$$
P=-\rho \dot{\phi}, \quad P=-\ddot{\varphi} .
$$

Accordingly, the coupling conditions expressing the continuity of the normal component of displacements and tractions at the fluid-solid interface can be written in 4 different ways:

1) $P-\mathbf{u}_{s}$ formulation

$$
\ddot{\mathbf{u}}_{s} \cdot \mathbf{n}_{s}=\frac{1}{\rho_{f}} \nabla P \cdot \mathbf{n}_{f}, \quad \boldsymbol{\sigma}_{s} \cdot \mathbf{n}_{s}=P \mathbf{n}_{f},
$$




\begin{tabular}{lcccc}
\hline & Size $\left(\mathrm{km}^{2}\right)$ & $V_{p}(\mathrm{~m} / \mathrm{s})$ & $V_{s}(\mathrm{~m} / \mathrm{s})$ & $\rho\left(\mathrm{kg} / \mathrm{m}^{3}\right)$ \\
\hline fluid & $26.4 \times 6$ & 1500 & 0 & 1000 \\
\hline solid & $26.4 \times 7.2$ & 3400 & 1963 & 2500 \\
\hline
\end{tabular}

Table 1 Physical parameters of the bilayered model used in the benchmark tests.

2) $\varphi-\mathbf{u}_{s}$ formulation

$$
\mathbf{u}_{s} \cdot \mathbf{n}_{s}=-\frac{1}{\rho_{f}} \nabla \varphi \cdot \mathbf{n}_{f}, \quad \boldsymbol{\sigma}_{s} \cdot \mathbf{n}_{s}=-\ddot{\varphi} \mathbf{n}_{f},
$$

3) $\phi-\mathbf{u}_{s}$ formulation

$$
\dot{\mathbf{u}}_{s} \cdot \mathbf{n}_{s}=-\nabla \phi \cdot \mathbf{n}_{f}, \quad \boldsymbol{\sigma}_{s} \cdot \mathbf{n}_{s}=-\rho_{f} \dot{\phi} \mathbf{n}_{f},
$$

4) $\mathbf{u}_{f}-\mathbf{u}_{s}$ formulation

$$
\mathbf{u}_{s} \cdot \mathbf{n}_{s}=-\mathbf{u}_{f} \cdot \mathbf{n}_{f}, \quad \boldsymbol{\sigma}_{s} \cdot \mathbf{n}_{s}=-\kappa\left(\nabla \cdot \mathbf{u}_{f}\right) \mathbf{n}_{f} .
$$

Note that the continuity of normal component of displacements are not strictly satisfied in cases $P-\mathbf{u}_{s}$ and $\phi-\mathbf{u}_{s}$ formulation, where only the normal component of acceleration and velocity are enforced to be continuous, respectively. We show how this affects the SEM results in the numerical tests.

Using SEM for the space discretization, these requirements need to be met in the weak forms of acousticand elastic-wave equation which are obtained by dotting with arbitrary test functions. We get 4 semidiscretized systems for representing the fluid-solid coupled modeling. For brevity, we write these systems in matrix form and categorize them according to the symmetry:

$P-\mathbf{u}_{s}$ system (non-symmetric)

$$
\left(\begin{array}{cc}
M_{f} & \mathbf{A} \\
\mathbf{0} & \mathbf{M}_{s}
\end{array}\right)\left(\begin{array}{c}
\ddot{P} \\
\ddot{\mathbf{u}}_{s}
\end{array}\right)+\left(\begin{array}{cc}
D_{f} & \mathbf{0} \\
\mathbf{0} & \mathbf{D}_{s}
\end{array}\right)\left(\begin{array}{c}
\dot{P} \\
\dot{\mathbf{u}}_{s}
\end{array}\right)+\left(\begin{array}{cc}
K_{f} & \mathbf{0} \\
\mathbf{A}^{\mathbf{T}} & \mathbf{K}_{s}
\end{array}\right)\left(\begin{array}{c}
P \\
\mathbf{u}_{s}
\end{array}\right)=\left(\begin{array}{c}
P_{f} \\
\mathbf{f}_{s}
\end{array}\right)
$$

$\varphi-\mathbf{u}_{s}$ system (non-symmetric)

$$
\left(\begin{array}{cc}
M_{f} & \mathbf{0} \\
\mathbf{A}^{\mathbf{T}} & \mathbf{M}_{s}
\end{array}\right)\left(\begin{array}{c}
\ddot{\varphi} \\
\ddot{\mathbf{u}}_{s}
\end{array}\right)+\left(\begin{array}{cc}
D_{f} & \mathbf{0} \\
\mathbf{0} & \mathbf{D}_{s}
\end{array}\right)\left(\begin{array}{c}
\dot{\varphi} \\
\dot{\mathbf{u}}_{s}
\end{array}\right)+\left(\begin{array}{cc}
K_{f} & \mathbf{A} \\
\mathbf{0} & \mathbf{K}_{s}
\end{array}\right)\left(\begin{array}{c}
\varphi \\
\mathbf{u}_{s}
\end{array}\right)=\left(\begin{array}{c}
\varphi_{f} \\
\mathbf{f}_{s}
\end{array}\right)
$$

$\phi-\mathbf{u}_{s}$ system (symmetric)

$$
\left(\begin{array}{cc}
M_{f} & \mathbf{0} \\
\mathbf{0} & \mathbf{M}_{s}
\end{array}\right)\left(\begin{array}{c}
\ddot{\phi} \\
\ddot{\mathbf{u}}_{s}
\end{array}\right)+\left(\begin{array}{cc}
D_{f} & \mathbf{A} \\
\mathbf{A}^{\mathbf{T}} & \mathbf{D}_{s}
\end{array}\right)\left(\begin{array}{c}
\dot{\phi} \\
\dot{\mathbf{u}}_{s}
\end{array}\right)+\left(\begin{array}{cc}
K_{f} & \mathbf{0} \\
\mathbf{0} & \mathbf{K}_{s}
\end{array}\right)\left(\begin{array}{c}
\phi \\
\mathbf{u}_{s}
\end{array}\right)=\left(\begin{array}{c}
\phi_{f} \\
\mathbf{f}_{s}
\end{array}\right)
$$

$\mathbf{u}_{f}-\mathbf{u}_{s}$ system (symmetric)

$$
\left(\begin{array}{cc}
\mathbf{M}_{f} & \mathbf{0} \\
\mathbf{0} & \mathbf{M}_{s}
\end{array}\right)\left(\begin{array}{c}
\ddot{\mathbf{u}}_{f} \\
\ddot{\mathbf{u}}_{s}
\end{array}\right)+\left(\begin{array}{cc}
\mathbf{D}_{f} & \mathbf{0} \\
\mathbf{0} & \mathbf{D}_{s}
\end{array}\right)\left(\begin{array}{c}
\dot{\mathbf{u}}_{f} \\
\dot{\mathbf{u}}_{s}
\end{array}\right)+\left(\begin{array}{cc}
\mathbf{K}_{f} & \mathbf{A} \\
\mathbf{A}^{\mathbf{T}} & \mathbf{K}_{s}
\end{array}\right)\left(\begin{array}{c}
\mathbf{u}_{f} \\
\mathbf{u}_{s}
\end{array}\right)=\left(\begin{array}{c}
\mathbf{f}_{f} \\
\mathbf{f}_{s}
\end{array}\right) .
$$

Here, $\mathbf{A}$ is the coupling matrix between the fluid and solid domain, and the remaining matrices correspond to conventional notations in SEM, namely, $M_{f}$ and $\mathbf{M}_{s}$ are the mass matrices, $D_{f}$ and $\mathbf{D}_{s}$ are the matrices for implementing absorbing boundary condition, and $K_{d}$ and $\mathbf{K}_{s}$ are the stiffness matrices.

\section{Numerical tests}

As shown above, only the $\mathbf{u}_{\mathbf{f}}-\mathbf{u}_{\mathbf{s}}$ system uses a vector-valued wave equation in the fluid domain. Therefore, it is less efficient than the other three using a scalar-valued equation in the fluid domain for seismic modeling. We here focus on the three systems where a scalar-valued equation is considered.

The validation tests are performed in 2D, and SEM results are benchmarked against the semi-analytical solution of bilayered model (Diaz and Ezziani, 2008). The physical parameters of this model are listed in Table 1. The upper part is acoustic, while the lower part is elastic. The Legendre polynomial degree is $N=5$. A total number of $520 \times 200=104000$ elements is used. An explosive pressure-source with a 10 $\mathrm{Hz}$ Ricker wavelet is applied $500 \mathrm{~m}$ above the interface. A combination of sponge layers and radiative boundary condition is used on all the edges of the model to attenuate outgoing waves (Trinh et al., 2019).

Figure 1a shows the spectra of source-time functions for systems of $P-\mathbf{u}_{s}, \phi-\mathbf{u}_{s}$ and $\varphi-\mathbf{u}_{s}$, where wavelets of $\phi$ and $\varphi$ are obtained by single and double time-integration of the pressure wavelet, respectively (Eq. (3)). The dominant frequencies for $\phi$ and $\varphi$ wavelet both shift towards low frequency. This means an increase of low-frequency component for both source-time functions, and Figure $1 \mathrm{~b}$ displays that the attenuation effectiveness of absorbing boundaries for wavefield energy gets weak in these two systems. However, the physical variable of interest in the fluid domain is pressure. As Eq. (5) 
(a)

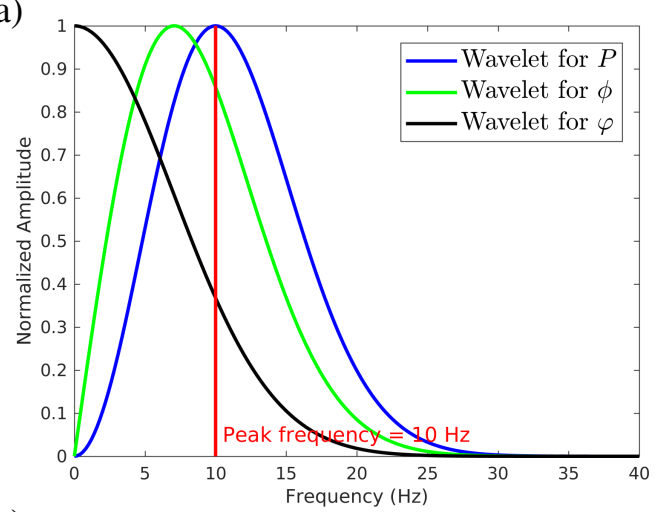

(c)

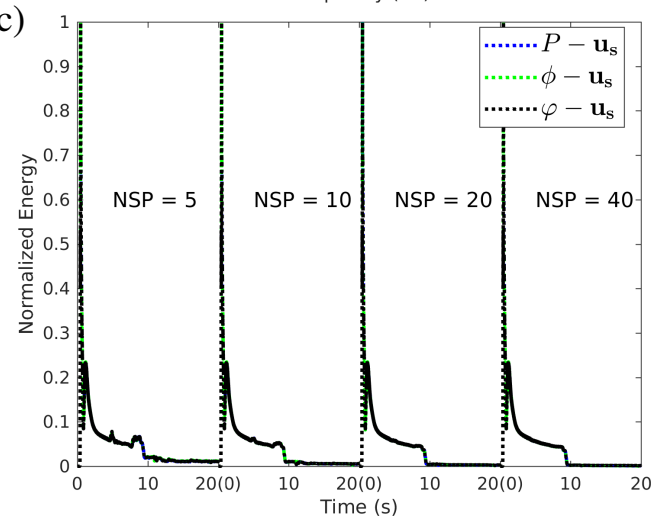

(b)

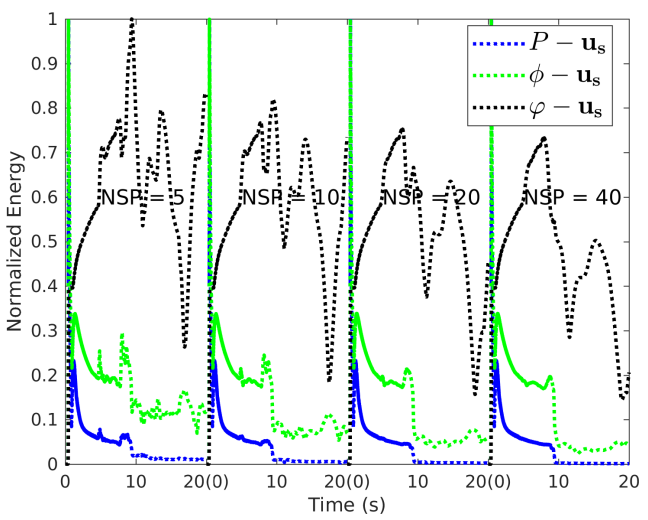

(d)

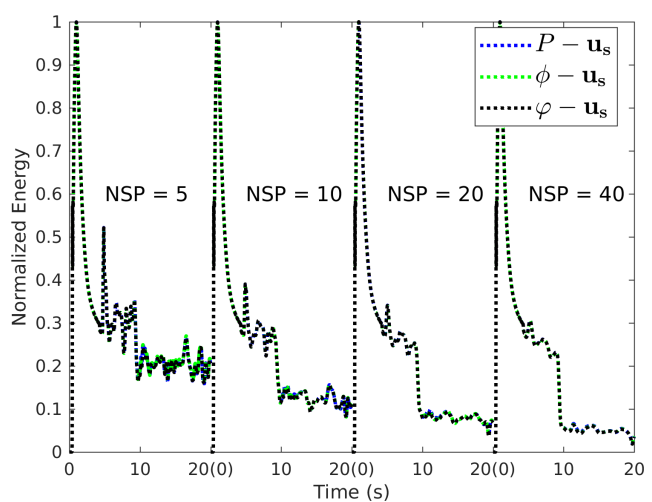

Figure 1 Evaluation of absorbing effectiveness for artificial boundary in $P-\mathbf{u}_{s}, \phi-\mathbf{u}_{s}$ and $\varphi-\mathbf{u}_{s}$ system. (a) Spectra of source-time function, (b) absorbing effectiveness of wavefield variables used in the acoustic-wave equation, (c) absorbing effectiveness of pressure in the fluid domain, (d) absorbing effectiveness of displacement in the solid domain. NSP: number of sponge elements.

shows, it can be obtained through first- or second-order derivation of potential variables, respectively, and we observe a similar attenuation effectiveness for the different systems in Figure 1c. Analogously, an attenuation comparison of wavefield energy in the solid domain is performed in Figure 1d to further demonstrate the absorbing consistency of different systems. Thus, a similar numerical accuracy for these three coupling systems is achieved (see Figure 2a). However, if a continuity check of displacement components is conducted at the fluid-solid interface (see Figure $2 \mathrm{~b}$ ), we will find that only the $\varphi-\mathbf{u}_{s}$ system produces the same vertical displacement component from both fluid and solid sides. This is because the other two systems enforce the normal component of acceleration and velocity to be continuous, respectively. Therefore, from the aspect of accuracy, $\varphi-\mathbf{u}_{s}$ system is a good choice for SEM modeling. In addition, the comparison in terms of elapsed time and memory consumption in Table 2 reveals a higher computational efficiency of $P-\mathbf{u}_{s}$ and $\varphi-\mathbf{u}_{s}$ system. The reason is that with non-symmetric coupling formulations $\left(P-\mathbf{u}_{s}\right.$ and $\left.\varphi-\mathbf{u}_{s}\right)$, the acoustic and elastic wave equations can be solved separately by explicit time-stepping schemes. On the contrary, the symmetric system $\phi-\mathbf{u}_{s}$ is implicit and needs to solve equations governing the fluid and solid domain simultaneously (Mönkölä, 2011). Here we use a staggered predictor-corrector iterative scheme (Komatitsch et al., 2000) for solving this $\phi-\mathbf{u}_{s}$ system which requires at least two iterations at each time step.

Finally, we show a 3D SEM implementation result of $\varphi-\mathbf{u}_{s}$ system in Figure 3. It is developed in the framework of SEM46 code using Cartesian-based mesh (Trinh et al., 2019). Here the fluid freesurface boundary condition and fluid-solid boundary condition are both considered, and we get the same modeling result as that produced by SPECFEM3D (Peter et al., 2011).

\section{Conclusions}

We have investigated 4 formulations for modeling seismic wave propagation in fluid-solid coupled media using the spectral element method. Their corresponding weak-form systems are presented and compared. In matrix form, the symmetry of $\phi-\mathbf{u}_{s}$ and $\mathbf{u}_{\mathbf{f}}-\mathbf{u}_{\mathbf{s}}$ system and non-symmetry of $P-\mathbf{u}_{s}$ and $\varphi-\mathbf{u}_{s}$ system are both revealed. Numerical tests indicate that the $\varphi-\mathbf{u}_{s}$ system has a better performance in terms of accuracy and computational efficiency in the SEM modeling than the other three, and the double integration of source-time function for displacement potential $(\varphi)$ will not decrease the 
(a)

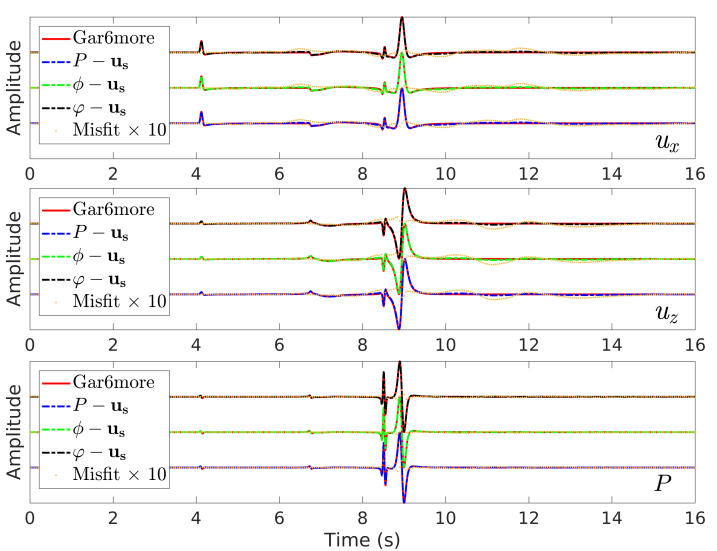

(b)

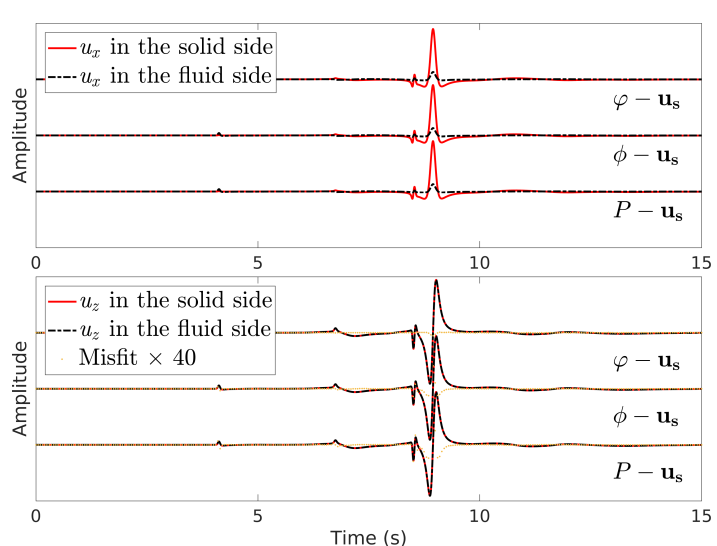

Figure 2 Validation for the modeling results of bilayered model. (a) Benchmark against the semi-analytical solution (Gar6more2D (Diaz and Ezziani] 2008)), (b) continuity check at the interface.

(a)

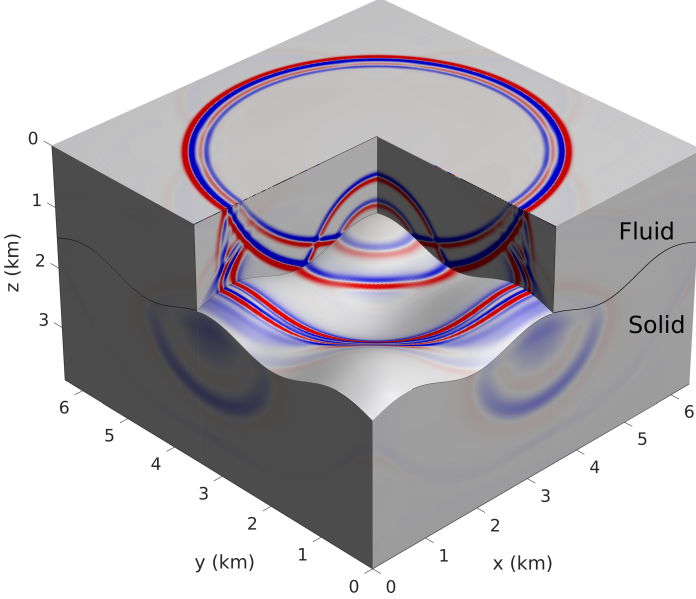

(b)
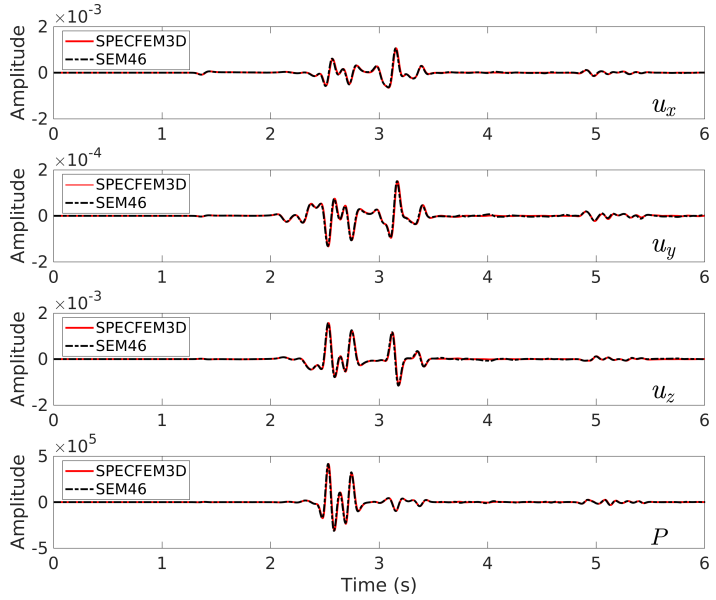

Figure 3 3D SEM results for fluid-solid coupled modeling in the framework of SEM46 code. (a) Snapshot of vertical displacement component, (b) seismogram comparison with results of SPECFEM3D (Peter et al. 2011). attenuation effectiveness of artificial boundary in modeling the physical wavefields of interest.

\section{Acknowledgements}

The research leading to these results has received funding from the European Union's Horizon 2020 research and innovation programme under the ENERXICO project, grant agreement No. 828947. This study was also partially funded by the SEISCOPE consortium (http://seiscope2.osug.fr), sponsored by AKERBP, CGG, CHEVRON, EQUINOR, EXXON-MOBIL, JGI, PETROBRAS, SCHLUMBERGER, SHELL, SINOPEC, SISPROBE and TOTAL. This study was granted access to the HPC resources of CIMENT infrastructure (https://ciment.ujfgrenoble.fr) and CINES/IDRIS/TGCC under the allocation 046091 made by GENCI.

\section{References}

Chaljub, E. and Valette, B. [2004] Spectral element modelling of three-dimensional wave propagation in a self-gravitating Earth with an arbitrarily stratified outer core. Geophysical Journal International, 158(1), 131-141.

Diaz, J. and Ezziani, A. [2008] Gar6more 2D. http://www.spice-rtn.org/library/software/Gar6more2D.

Everstine, G.C. [1997] Finite element formulatons of structural acoustics problems. Computers \& Structures, 65(3), 307-321.

Feng, X. [2000] Analysis of finite element methods and domain decomposition algorithms for a fluid-solid interaction problem. SIAM Journal on Numerical Analysis, 38(4), 1312-1336.

Komatitsch, D. [1997] Méthodes spectrales et éléments spectraux pour l'équation de l'élastodynamique $2 \mathrm{D}$ et $3 \mathrm{D}$ en milieu hétérogène. Ph.D. thesis, Institut de Géophysique du Globe de Paris.

Komatitsch, D., Barnes, C. and Tromp, J. [2000] Wave propagation near a fluid-solid interface: a spectral element approach. Geophysics, 65(2), 623-631.

Mönkölä, S. [2011] Numerical simulation of fluid-structure interaction between acoustic and elastic waves. Ph.D. thesis, University of Jyväskylä.

Peter, D., Komatitsch, D., Luo, Y., Martin, R., Le Goff, N., Casarotti, E., Le Loher, P., Magnoni, F., Liu, Q., Blitz, C., Nissen-Meyer, T., Basini, P. and Tromp, J. [2011] Forward and adjoint simulations of seismic wave propagation on fully unstructured hexahedral meshes. Geophysical Journal International, 186(2), 721-739.

Ross, M.R., Sprague, M.A., Felippa, C.A. and Park, K.C. [2009] Treatment of acoustic fluid-structure interaction by localized Lagrange multipliers and comparison to alternative interface-coupling methods. Computer Methods in Applied Mechanics and Engineering, 198(9-12), 986-1005.

Trinh, P.T., Brossier, R., Métivier, L., Tavard, L. and Virieux, J. [2019] Efficient 3D time-domain elastic and viscoelastic Full Waveform Inversion using a spectral-element method on flexible Cartesian-based mesh. Geophysics, 84(1), R75-R97. 